\title{
An Ocean Sensor for Measuring the Seawater Electrochemical Response of 8 Metals Referenced to Zinc, for Determining Ocean $\mathrm{pH}$.
}

\author{
Martin Brooke ${ }^{1}$, Eli Cole ${ }^{1}$, Julian Dale ${ }^{3}$, Anshuman \\ Prasad $^{1}$, Henry Quach ${ }^{2}$, Becca Bau ${ }^{1}$, and Douglas \\ Nowacek $^{1,3}$ \\ (1) Dept. of ECE, (2) Dept. of MEMS \\ and (3) Duke Marine Lab \\ Duke University \\ Durham, NC USA
}

\author{
Eeshan Bhatt \\ MIT-WHOI Joint Program \\ Woods Hole, MA USA
}

\begin{abstract}
We describe the use of a multi-metal electrochemical cell for measuring ocean $\mathrm{pH}$. The sensor was designed to be robust, inexpensive, and capable of 0.02 sensitivity to $\mathrm{pH}$ in the narrow ranges required for marine $\mathrm{pH}$ monitoring. A prototype sensor has undergone an extended ocean deployment with promising results.
\end{abstract}

Ocean Sensors; pH Sensors; Metal electrochemistry; Arduino.

\section{TECHNOLOGY APPROACH}

The idea behind the sensor comes from the very low cost unpowered sensors used for soil $\mathrm{pH}$ measurements [1]. These simple meters use two metals to generate a potential that is read on an analog meter and are surprisingly accurate at measuring soil $\mathrm{pH}$. We purchased and tested many of these sensors and were very impressed with the accuracy and repeatability of this simple technology. Based on this idea we measured the potential across Zinc and Copper in $\mathrm{pH}$ buffered solutions of Instant Ocean. We found that we could calibrate the electrochemical cell to approximately $0.02 \mathrm{pH}$ for the $\mathrm{pH} 7-9$ range. This led to the idea that we could use metals for a useful low cost ocean $\mathrm{pH}$ sensor.

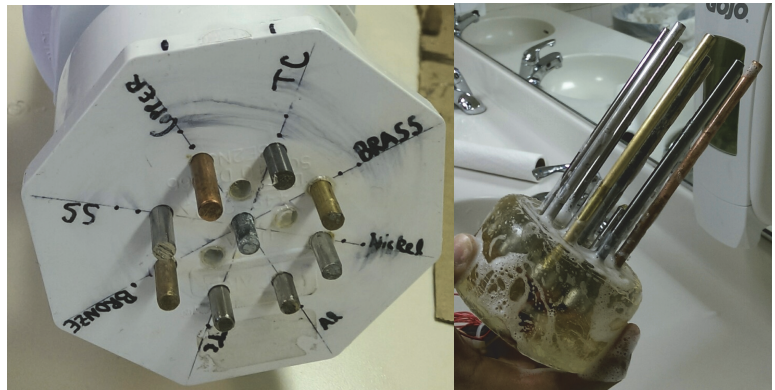

Figure 1. The nine metals used: 316 Stainless Steel; Copper; Tungsten Carbide; Brass; Nickel; Aluminium; Titanium; Bronze; and Zinc (in the center). A PVC mold was use to cast the metals in epoxy for a water tight seal.

Biofouling and corrosion will alter the metal surfaces over time [2], and the metal surface potentials will be sensitive to chemical potentials other than just $\mathrm{pH}$. To overcome this we propose using metal diversity. We are currently using 9 different $1 / 4$ inch metal rods in our prototype sensor (Figure 1). These are all mass produced and available at low cost.

\section{SENSOR BODY DESIGN}

The overall sensor form factor is based on a low cost 4" diameter $\mathrm{x} 1 \mathrm{~m}$ long PVC tube housing. PVC is an adequate material for shallow water, and can act as a mold for an epoxy encased sensor package that will survive greater water depths. A significant advantage of the multi-metal sensor proposed is that it is very robust and has no moving parts. Hence it can be encased in epoxy and expected to survive the high pressures associated with ocean depths.

\section{SENSOR DESIGN AND CONSTRUCTION}

The sensor measures the electrochemical response of 8 different metals referenced to Zinc and 3 ocean temperature probes. The measurements and a real time clock reading are saved in nonvolatile memory and retrieved via a USB cable when the sensor is retrieved. These data can be converted to $\mathrm{pH}$ using a calibration table. We hope that salinity and other properties might be extracted from the data using multivariate analysis.

\section{A. Electrochemical Potential Measurement System}

The measurements are carried out using an Arduino UNO R3 with a wireless SD shield (Figure 2). The electrochemical voltages are measured using two ADS1115 16-Bit ADC - 4 Channel with Programmable Gain Amplifier breakout boards from Adafruit. The temperature of the ocean is measured using three redundant Vktech DS18b20 Waterproof Temperature Sensors. The measurement time is kept using a SainSmart I2C RTC DS1307 AT24C32 Real Time Clock. The voltage temperature and time data is stored on a Kingston $8 \mathrm{~GB}$ microSDHC Class 4 Flash Memory Card. This memory card can store approximately 20,000 sets of measurements.

\section{B. Low Power Operation}

To achieve very low power operation the sensor uses an ATtiny85 microcontroller (programmed using the Arduino IDE). The ATtiny 85 can be put to sleep and in this state uses 

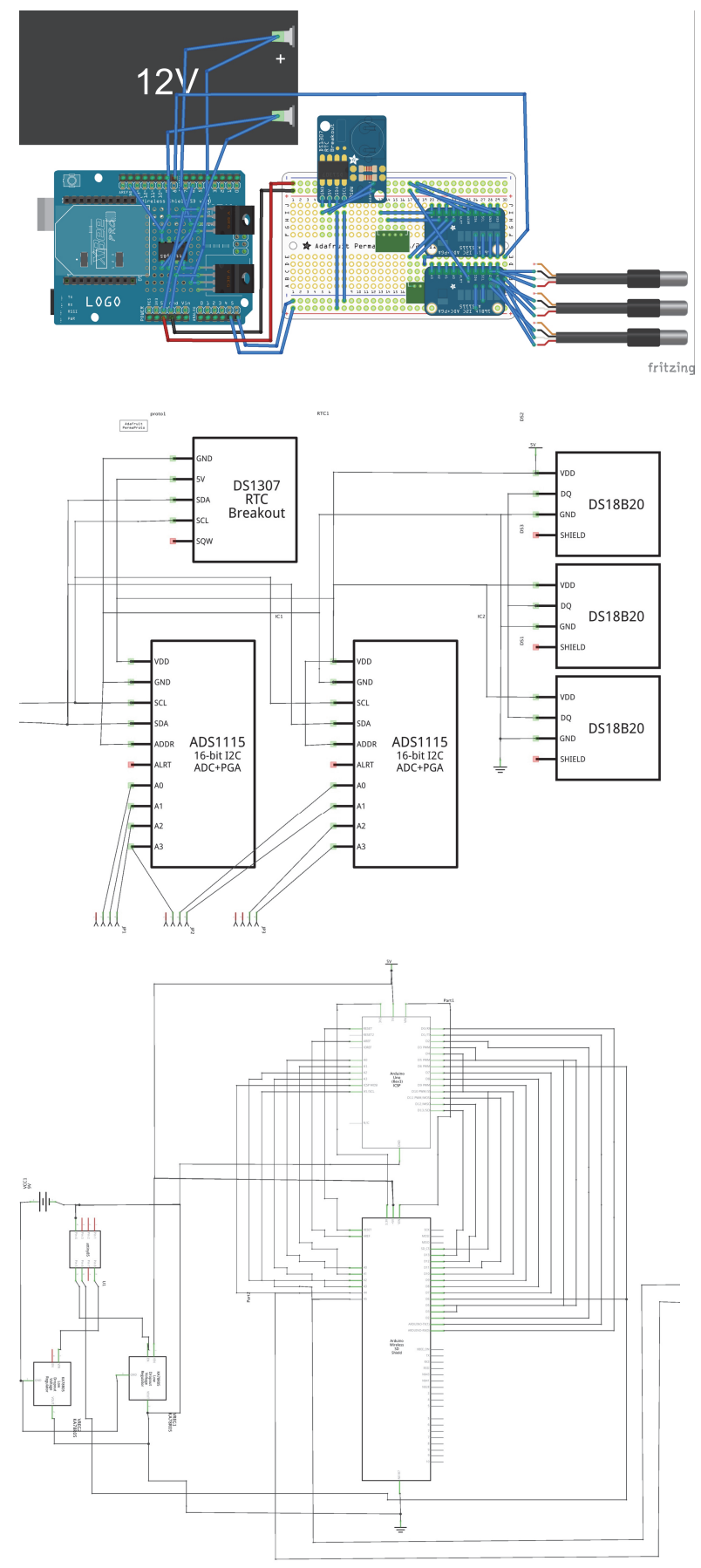

Figure 2. The Electrochemical Potential Measurement System. A drawing of the parts used to assemble the system (top). A circuit diagram of the modules used for measurement (middle), and the Arduino board, shield, and power supply control ATtiny 85 system (bottom).

less than $6 \mathrm{uA}$ of supply current. To power the ATtiny 85 and the main Arduino processor a pair of efficient D24V5F5 Pololu 5V, 500mA Step-Down Voltage Regulators are used operating from $12 \mathrm{~V}$ batteries. These synchronous buck voltage regulators use less than 200uA of current to operate. The main measurement system is turned on and off by the ATtiny85, so the high power used by the Arduino and other devices is only drawn for as long as is necessary to take measurements of the electrochemical voltages and other readings (about 5 seconds). The more than 3 Amp hours provided by the 12 Volt Lead Acid batteries then allow for more than a year of operation with the $200 \mathrm{uA}$ current draw of only the ATtiny 85 . With a $2.2 \%$ duty cycle, the 40 milliamp current draw of the Arduino system (on the $12 \mathrm{~V}$ battery) will last more than 77 days. A $2.2 \%$ duty cycle allows a 5 second reading to occur roughly every 4 minutes. We chose a 5 min reading cycle and so achieve more than 77 days of life from one battery charge.

\section{Cost Estimate}

The estimated small quantity cost, including shipping, of the electronics is less than 200 USD in 2015 [3]. The hardware used to make the sensor body also cost less than 200 USD. The metals used for the sensor head cost less than 50 USD giving a total build cost of $<450$ USD. The sensor electronics and body could be repurposed to other ocean sensor applications, with the addition of a sensor package, making a 400 USD sensor platform (not including the sensor) feasible for anyone able to assemble the electronics and casing.

\section{Software}

The full system software is available online [4]. This code was developed using open-source packages provided by the vendors of the hardware and by the Arduino online community.

\section{SEnsor Test Results}

The sensor was deployed in a seawater tank at the Seattle Aquarium as part of the Wendy Schmitt Ocean XPRIZE contest [4]. The raw sensor data taken over one month (approx. 7000 readings) are presented in Figure 3. Seawater $\mathrm{pH}$ measured by the XPRIZE validation team using bench-top spectrophotometry appears in Figure 4 plotted against the date of the measurements.

\section{TEST RESUlts Discussion}

While it does appear that the sensor is operational, there are many issues yet to explore for the sensor to be used to routinely measure ocean $\mathrm{pH}$. The time spent in the ocean was surprisingly lenient on the metal sensor rods. Figure 5. Shows the state of the rods after the Seattle trials. However it is clear from the measurements that the rod voltages do seem to stabilize over time despite the continued changes observed in $\mathrm{pH}$. We suspect that the rods are being inactivated by a combination of corrosive coatings and biofouling. 


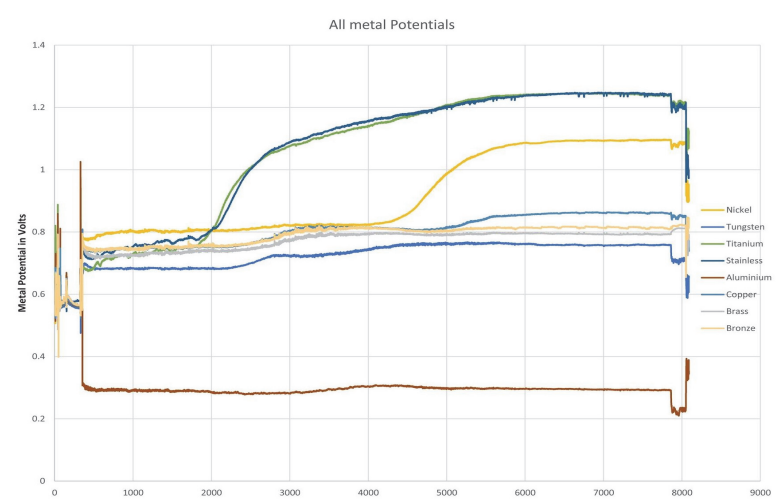

Figure 3. The measured electrochemical response of the 8 metals referenced to Zinc. There are 8000 measurements plotted. The time the sensor was out of the water is visible at the beginning and end of the data.

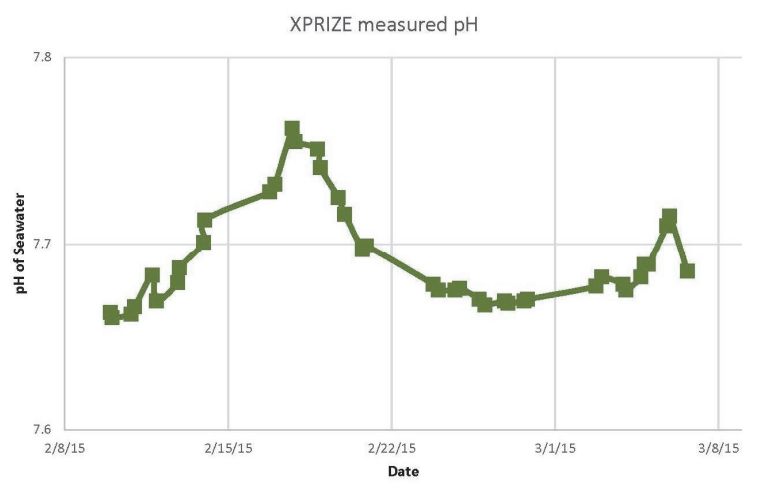

Figure 4. Seawater $\mathrm{pH}$ measured by Spectrophotometry versus date. The samples span approximately one month by the XPRIZE validation team .

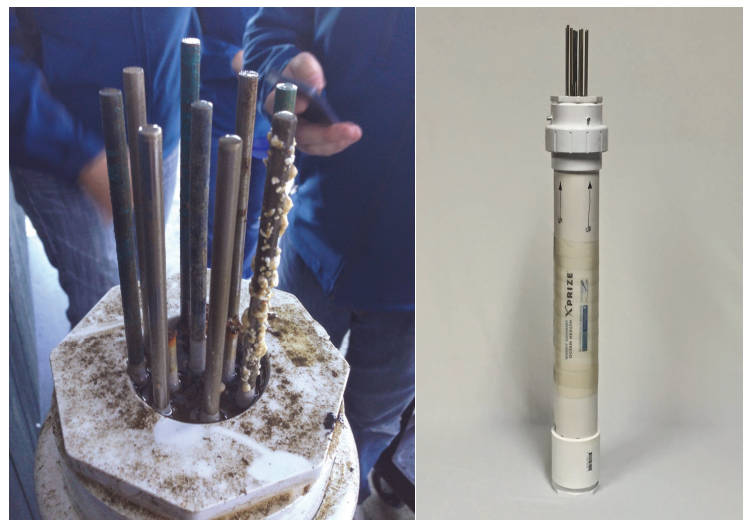

Figure 5. The physical condition of the sensor rods after the Seattle seawater trails (left). Biofouling affected the Nickel rod considerably more than the other metals. The completed sensor (right).

\section{Multivariate Neural Network PH Estimation}

In order to determine the $\mathrm{pH}$ of the seawater we propose to use a multivariate estimation from all of the sensor data. Since we expect that the metal electrochemical response will be time dependent due to corrosion and biofouling, time dependent neural networks that learn to predict the $\mathrm{pH}$ from past sensor behavior, seem a likely candidate. To test the viability of this approach we experimented with training time dependent neural networks on our measurement data.

Figure 6 is a schematic of a neural network implemented in MATLAB [5]. The network input is a combination of the time of the measurement, and the electrochemical voltage response from each of the eight metals with respect to Zinc. The current input and the most recent past 5 inputs are normalized to the minimum and maximum of the data, weighted, biased, and summed to produce 3 hidden values. These hidden values are put through a sigmoid nonlinearity, weighted and biased, before being summed linearly to produce the $\mathrm{pH}$ estimate.

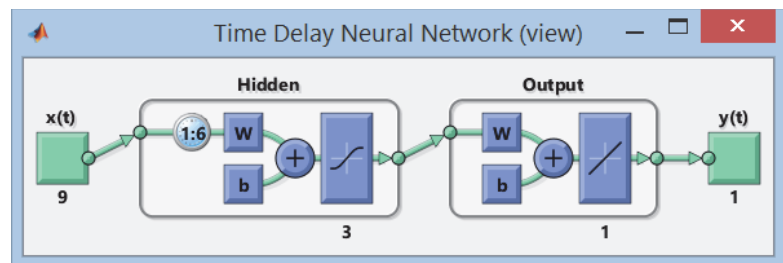

Figure 6. Neural network architecture used to compute $\mathrm{pH}$ from the measured electrochemical response of the 8 metals referenced to Zinc and the sampling time.

When this network is trained using combinations of random weight selection and the Levenberg-Marquardt algorithm [6], we can obtain a set of weights that appear in Figure 7 and Appendix I. We can see that the first input (time), and the seventh and eighth inputs (Stainless and Titanium) have the largest weights. But some contribution is used from all the metals, with Aluminum (the second input) having the lowest weight magnitudes.

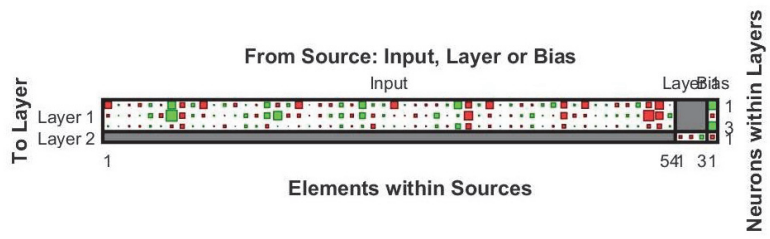

Figure 7. After training, the weights obtained are represented graphically in here. Red is negative green is positive, and square size represents magitude. The actual weights are in appendix I.

The performance of this network is presented in Figure 8 . The network was trained on the first 3000 samples after the sensor was placed in the water, and so performs very well on this data. The network was tested on samples 3001 to 7000 . The performance on the testing data is not as good as on the training data however, predicted measurements match to within $0.02 \mathrm{pH}$ until near the end of the data set. We suspect the metals in the sensor were too badly coated with corrosion and biofouling to 
function after about 3 weeks and so did not register the final $\mathrm{pH}$ spike in the XPRIZE data.

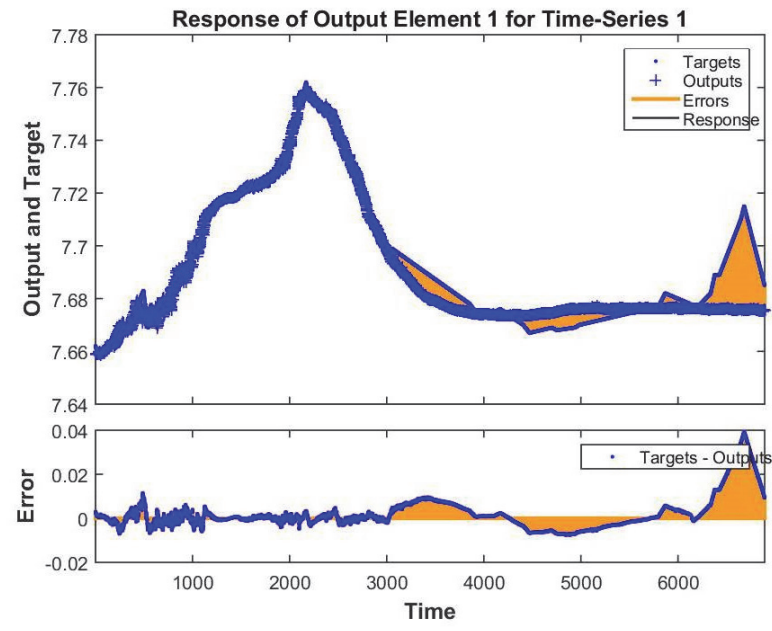

Figure 8. Neural network predicted $\mathrm{pH}$ computed from the measured electrochemical response of the 8 metals referenced to Zinc.versus sample number. The samples span approximately one month. The samples from 0-3000 were used to train the neural netowrk, the values from 3001-7000 were predicted.

\section{ACKNOWLEDGMENTS}

The Department of Electrical and Computer Engineering at Duke University provided funding for this project. All work was done at Duke University. The XPRIZE foundation provided access to the test facilities, and provided their measurements of $\mathrm{pH}$, temperature and salinity taken during the Seattle trials. The entire Fall 2014 Ocean Engineering class at Duke University helped with the sensor fabrication. In particular the authors thank Ryan Yoon, Abhishek Ghimire, and Rahul Harikrishnan for their help.
Appendix I. The neural network weights for the data presented in Figure 8.

$$
\begin{aligned}
& \begin{array}{l}
\text { Layer } 1 \text { weights } \\
\text { Columns } 1 \text { thro }
\end{array} \\
& \begin{array}{llllllll}
-3.1387 & 0.0885 & -0.1536 & -0.5287 & 0.4423 & -0.1179 & 3.7617 & -1.4839
\end{array} \\
& \begin{array}{rrrrrrrr}
-0.3955 & 0.0046 & -0.1227 & 0.0147 & 0.9936 & -0.8162 & 7.6364 & -1.8478 \\
0.0676 & 0.0125 & -0.1152 & -0.1335 & 0.1665 & 0.0582 & -0.4034 & -1.0224 \\
\hline
\end{array} \\
& \begin{array}{llllllll}
\text { Columns } 9 & \text { through } 16 & 0.0456 & 0.3810 & -0.5954 & 0.0367 & -0.1069 & 1.7901 \\
0.6486 & -3.2811 & 0.0456 & -0.0224 & 0.0010 & 0.9074 & -0.4285 & -0.5990
\end{array} \\
& \begin{array}{lllllllll}
0.2771 & -0.4177 & 0.0600 & -0.0224 & 0.0691 & 0.7914 & -0.4401 & 1.9549
\end{array}
\end{aligned}
$$

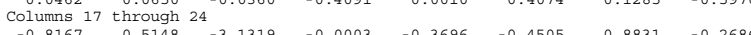

$$
\begin{aligned}
& \begin{array}{lllllllll}
-0.8167 & 0.5148 & -3.1319 & -0.0003 & -0.3696 & -0.4505 & 0.8831 & -0.2686
\end{array} \\
& \begin{array}{lrrrrrrr}
4.4373 & -0.5598 & -0.6066 & -0.0184 & -0.3637 & 0.3711 & 0.9786 & -0.5429 \\
0.0127 & 0.0005 & -0.0735 & 0.0323 & -0.1390 & -0.0791 & 0.3573 & -0.0918
\end{array} \\
& -0.2416-0.250 \\
& \begin{array}{rrrrrrrr}
2.9174 & 0.3276 & 0.2732 & -3.2565 & 0.0500 & -0.1673 & -0.2416 & -0.2503 \\
2.0188 & -0.5764 & 0.2422 & -0.0627 & -0.0268 & -0.5899 & -0.0713 & 1.4908 \\
0.2678 & -0.9899 & 0.0568 & 0.0668 & -0.0137 & -0.1390 & -0.0376 & 0.4210 \\
0
\end{array} \\
& \text { Columns } 33 \text { through } 40 \\
& \begin{array}{rrrrrrrr}
0.2270 & 3.4961 & -2.2329 & 0.2539 & -3.4003 & 0.0502 & 0.2418 & -0.3801 \\
-0.0135 & 0.2572 & -4.5296 & 0.0663 & -0.4989 & 0.0369 & -0.1571 & 0.2181
\end{array} \\
& \begin{array}{llllllll}
-0.1373 & 1.4940 & -1.6798 & 0.0689 & -0.3183 & 0.0207 & -0.1802 & -0.1375
\end{array} \\
& \begin{array}{llllllll}
\text { Columns } 41 & \text { through } & 48 & & & & & \\
-0.2108 & 0.3612 & 1.2575 & -2.2115 & 0.1826 & -2.8735 & 0.1005 & 0.1347
\end{array} \\
& \begin{array}{rrrrrrrr}
-0.2108 & 0.3612 & 1.2575 & -2.2115 & 0.1826 & -2.8735 & 0.1005 & 0.1347 \\
0.9052 & 0.0338 & -0.0398 & -2.3975 & -0.5496 & -0.3452 & 0.0377 & 0.0939 \\
0.3599 & 0.0156 & -0.1662 & -0.7728 & 0.0202 & -0.1852 & 0.0516 & -0.0270
\end{array} \\
& \begin{array}{llllll}
-0.7388 & -0.5018 & 1.0679 & -1.7419 & -4.3949 & 0.0080
\end{array} \\
& \begin{array}{rrrrrr}
-0.7388 & -0.5018 & 1.0679 & -1.7419 & -4.3949 & 0.0080 \\
0.1882 & 0.3168 & 0.1616 & -6.9339 & -3.5615 & 0.8280 \\
-0.1691 & 0.1227 & 0.0704 & -0.7157 & -1.2038 & 0.1049
\end{array} \\
& \begin{array}{ll}
\text { Layer } 2 \text { weights } \\
-0.4772-0.5960 \quad 0.9230
\end{array} \\
& \text { Layer } 1 \text { bias } \\
& \begin{array}{r}
5.2704 \\
-0.6996 \\
5.0804
\end{array} \\
& \text { Layer } 2 \text { bias }
\end{aligned}
$$

\section{REFERENCES}

[1] 3-in-1 Moisture Meter with Light \& PH Test Function, \$4.30 Amazon Date: $7 / 30 / 14$.

[2] Dexter, Stephen C., and John P. LaFontaine. Effect of natural marine biofilms on galvanic corrosion predicted using potentiodynamic polarization curves. No. CONF-980316--. NACE International, Houston, TX (United States), 1998.

[3] Google Spreadsheet with quatitiy, purchase links, and current cost of all parts: http://bit.ly/1cM0eVC

[4] https://hackaday.io/project/4122-ocean-xprize-ph-sensor/

[5] MATLAB R2015a, The MathWorks Inc., Natick, MA, USA.

[6] Moré, Jorge J. "The Levenberg-Marquardt algorithm: implementation and theory." Numerical analysis. Springer Berlin Heidelberg, 1978. 105-116. 